\title{
A new cyano-substituted fluorescamine superior to its original form as a fluorescent probe for amino acid detection
}

Jiro Motoyoshiya, ${ }^{*}$ Satoshi Tomioka, Daigo Kobayashi, Tetsuya Fujimoto

Course of Applied Molecular Chemistry, Department of Chemistry and Materials, Faculty of Textile Science and Technology, Shinshu University, 3-15-1 Tokida, Ueda, Nagano, 386-8567, Japan

* Corresponding author, Tel.: +81 268 5402; fax: +81 268 5391;

E-mail address: jmotoyo@shinshu-u.ac.jp

Keywords:

fluorescamine

fluorescent probe

cyano group

peroxyoxalate chemiluminescence

\begin{abstract}
Synthesis and spectral study of two new cyano-substituted fluorescamine as the fluorescent probes for amino acid detection have been carried out comparing with the original fluorescamine. Of the three compounds, the derivative with a cyano group at the mata-position on the 4-phenyl group was found to be superior to the original one in the reactivity toward some amino acids as well as the fluorescence intensity of the adducts. The fluorescent amino acid adducts were also applied to the peroxyoxalate chemiluminescence system as the fluorophores, in which the derivative described above was found to be more effective also in chemiluminescence than the original one.
\end{abstract}


Fluorescamine (1), 4-phenylspiro[furan-2(3H),1'-phtalan]-3,3'-dione, is a commercially available fluorescent probe for the detection of primary amines including biologically important compounds, especially, for the analysis of amino acids and proteins. ${ }^{1}$ In spite of prosperous exploitation of various fluorescent probes for amino acid detection, ${ }^{2}$ fluorescamine is still keeping much importance and convenience in biochemical analysis. ${ }^{3}$ Non-fluorescent 1 reacts with primary amines smoothly to produce the adducts, the strongly fluorescent pyrrolinones (Scheme 1). Needless to say, the amino acid determination by HPLC using $\mathbf{1}$ is a routine technique in the field of biological chemistry, which enables the detection of the picomole level of amino acids. ${ }^{4}$ Because of its prominent utility, several fluorescamine derivatives with alkoxy and halogen substituents on the 4-phenyl group have been prepared and their properties as the fluorescent probe investigated up to date, ${ }^{5}$ but more excellent one than the original fluorescamine has not been reported yet. Due to expectation that the introduction of the electron-withdrawing functionality on the 4-phenyl group might improve the required property as the fluorescent probe, we prepared two new fluorescamine derivatives $\mathbf{2}$ and $\mathbf{3}$ with cyano groups on the 4-phenyl group and found that the derivative with a cyano group on the meta-position of 4-phenyl groups was superior to fluorescamine (1) in the reactivity toward some amino acids as well as the fluorescence intensities of the amino acid adducts.

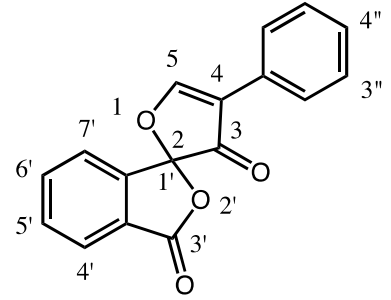

Fluorescamine (1)

non-fluorescent

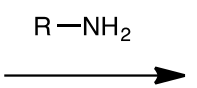<smiles>O=C(O)c1ccccc1C1(O)C(=O)C(c2ccccc2)=CN1P</smiles>

strongly fluorescent

Scheme 1. Reaction of fluorescamine (1) with primary amines forming the fluorescent adducts.

The fluorescamine derivatives $2(\mathrm{R}=3$ "- $\mathrm{CN})$ and $3(\mathrm{R}=4$ "-CN) were synthesized according to the general method for the synthesis of 1 using $m$ - or $p$-cyanobenzaldehydes instead of benzaldehyde, ${ }^{6}$ respectively, as shown in Scheme 2. Starting from the known 
2-benzylidene-1,3-indandiones with the cyano substituents on the phenyl groups of the benzylidene moieties, ${ }^{7}$ the sequence processes, an epoxidation with alkaline hydrogen peroxide and the ring cleavage affording the enol of the $\alpha$-diketones followed by construction of spiro-compounds using $N, N$-dimethylformamideacetal, finally gave the 3"- and 4"-cyano substituted fluorescamines $\mathbf{2}$ and $\mathbf{3}$. The new compounds $\mathbf{2}$ and $\mathbf{3}$ were identified by IR, NMR, and HRMS as shown in the experimental section (Supporting Information).
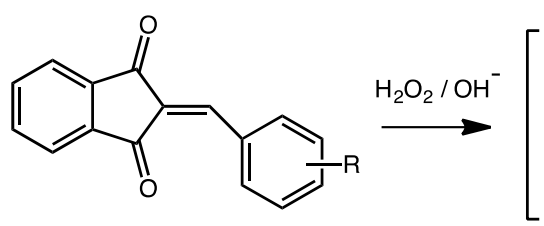<smiles>O=C1c2ccccc2C(=O)C12OC2c1ccccc1</smiles><smiles>O=C(O)c1ccccc1C(=O)C(O)=Cc1ccccc1</smiles>
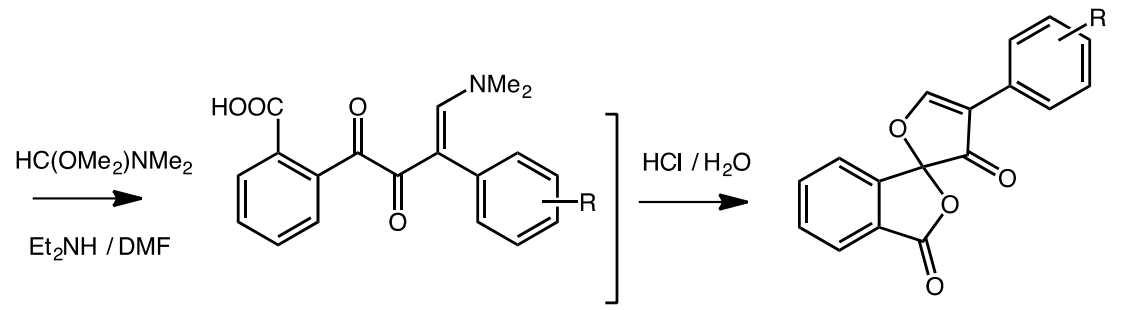

2: $\mathrm{R}=3^{\prime \prime}-\mathrm{CN}$

3: $\mathrm{R}=4^{\prime \prime}-\mathrm{CN}$

Scheme 2. Synthetic scheme of the fluorescamine derivatives 2 and $\mathbf{3}$.

Upon the reactions of the compounds 2, 3, and fluorescamine (1) with 3 equiv. of phenylalanine in an aqueous THF (THF: $\mathrm{H}_{2} \mathrm{O}=1: 1$ ) under the neutral conditions, the solution became fluorescent due to the formation of pyrrolinone adducts. The measurements of the fluorescence spectral change of the reaction mixtures revealed that the fluorescence intensity increased with the elapse of time in all cases as shown in Fig. 1a-c. The emission maxima of the phenylalanine adducts of 2 and 3 were observed at $464 \mathrm{~nm}$ and $467 \mathrm{~nm}$, respectively, which were blue-shifted by ca. $10 \mathrm{~nm}$ compared to that of 1 emitting at $476 \mathrm{~nm} .{ }^{8}$ In addition, the differences in the fluorescence intensity and the reactivity were also found, namely, the fluorescence intensity of the adduct of $\mathbf{2}$ was stronger than that of $\mathbf{1}$, but $\mathbf{3}$ was weaker than $\mathbf{1}$ at first glance. The plots of the increasing intensities against time gave the curves as shown in Fig. 1d. The kinetics of the reaction of 1 and alanine has been previously described as a sequential process, ${ }^{9}$ the Michael addition of the amino acid producing the intermediate and the following reconstruction affording the final fluorescent product, but the whole reaction can be approximated as a bimolecular process if only the initial stage of the reaction is cut off for comparison of the kinetic rates, because the 
latter reaction process was reported to be much faster than the former Michael addition. Therefore, the curves in Fig. 1d were kinetically treated by means of the bimolecular manner. As expected, the plots of the values containing the ratios of the product and probe concentrations, determined from each fluorescence intensities of the adducts described in Fig.1d, against time gave the almost linear lines, whose slopes led to estimation of the reaction rate constants, namely, $0.87,1.87$, and $3.80 \mathrm{M}^{-1} \mathrm{sec}^{-1}$ for the reactions of phenylalanine with $\mathbf{1}, \mathbf{2}$, and $\mathbf{3}$, respectively. The ratio of the rate constants for $\mathbf{1}, \mathbf{2}$, and $\mathbf{3}$ was calculated to be approximately 1: $2.15: 4.37$, respectively, indicating that the introduction of the cyano groups, the electron-withdrawing substituents, enhanced the reactivity toward phenylalanine, in which the cyano groups would be effective to reduce the electron density at 5-position of $\mathbf{2}$ and $\mathbf{3}$, making the Michel addition of phenylalanine favorable.

(a) $1+$ phenylalanine

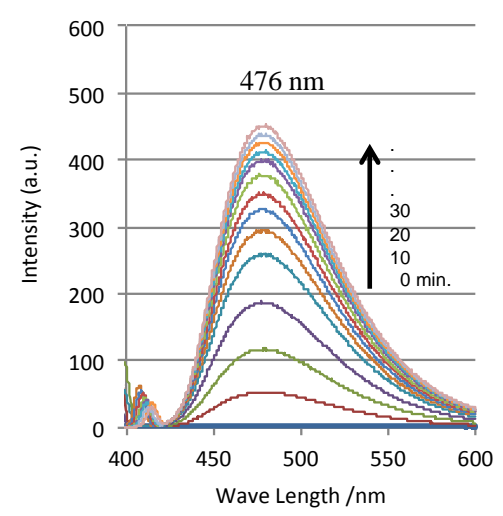

(d)

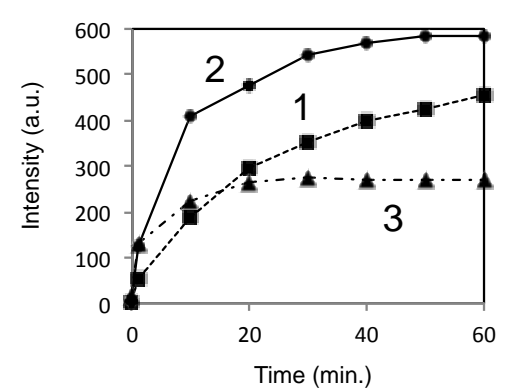

(b) $2+$ phenylalanine

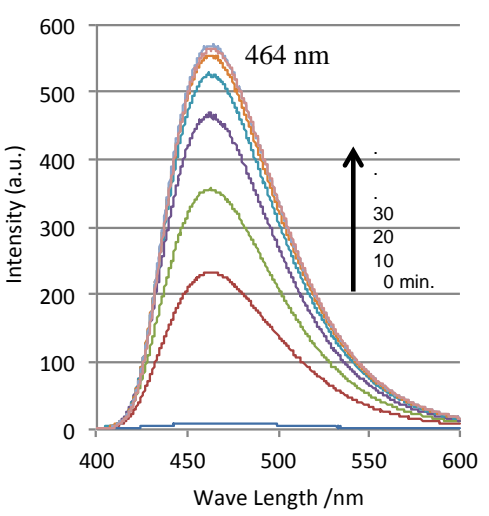

Fig. 1. Fluorescence spectral change in the reaction of 1, 2, and $\mathbf{3}$ with phenylalanine and kinetic analysis. (a), (b), and (c) for the reaction of 1, 2, and 3 with phenylalanine, $[1]=[2]=[3]=[$ phenylalanine $]=2 \mathrm{x}$ $10^{-3} \mathrm{M}$ in $\mathrm{THF} / \mathrm{H}_{2} \mathrm{O}$ (1:1). (c) Time course of spectral change measured by the fluorescence spectra of the adducts. (e) Kinetic curves by the treatment as the bimolecular process of the reactions of phenylalanine and $\mathbf{1}, \mathbf{2}$, and $\mathbf{3}$. The rates were estimated as $0.87 \mathrm{M}^{-1} \mathrm{sec}^{-1}, 1.87 \mathrm{M}^{-1} \mathrm{sec}^{-1}$, and $3.80 \mathrm{M}^{-1} \mathrm{sec}^{-1}$ for $\mathbf{1}, \mathbf{2}$, and $\mathbf{3}$, respectively. (c) $3+$ phenylalanine

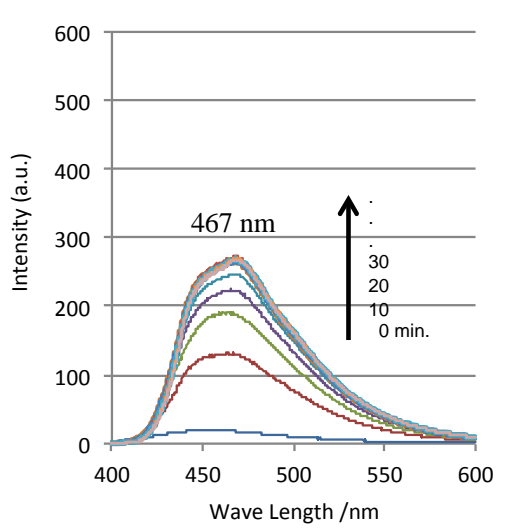

(e)

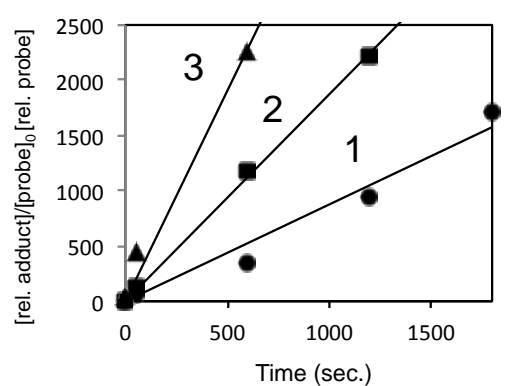


Since the most required performance for a fluorescent probe is the stronger fluorescence intensity in view of amino acid detection, 2 superior to the others as described above was used for the present investigation hereafter. It is known that the fluorescence intensity of the fluorescamine-amino acid adduct is dependent on the kind of amino acids, and various fluorescence quantum yields for several adducts of the amino acids were reported. For example, the values for phenylalanine, glycine, and alanine were 0.11, 0.092, and 0.081, respectively, namely, the ratio is 1: $0.84: 0.74 .{ }^{10}$ The fluorescence intensities in the reaction of 2 and some amino acids and bovine serum albumin (BSA) was compared to those of 1 (Fig. 2a), indicating that the adducts of $\mathbf{2}$ gave stronger intensities than those of $\mathbf{1}$ in all cases using several amino acids. The tendency of the fluorescence intensities of $\mathbf{2}$ and amino acids adducts, however, did not agree with that reported for $\mathbf{1}$, because the order of the intensity of $\mathbf{2}$ and three amino acids was, glycine, phenylalanine, and alanine, whose relative fluorescence quantum yields were $1: 1.13$ : 0.79 , respectively. On the other hand, the reaction profile of $\mathbf{1}$ and $\mathbf{2}$ and glycine was described in Fig. $2 \mathrm{~b}$ by plotting the fluorescence intensity against the glycine concentration, showing that the reaction of 2 completed with almost 1 eqiv. glycine similarly to $\mathbf{1}$. As shown in Fig. $2 \mathrm{~b}$ the sensitivity for detection of glycine by using $\mathbf{2}$ is at $\square \square \square \square$ omole level under the present conditions, but it will be possible to increase sensitivity at much higher level because analysis with 1 at the picomole level applying a column chromatography attached by a fluoromicrophotometer was reported. ${ }^{4}$

(a)

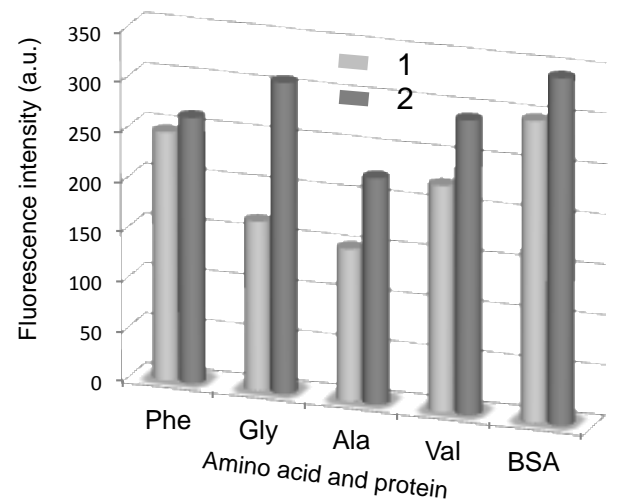

(b)

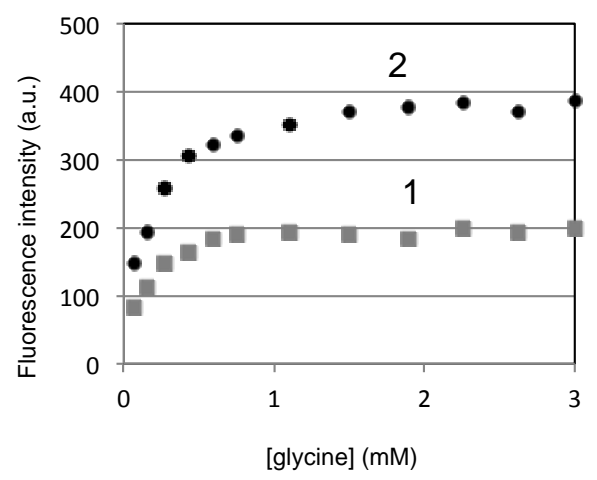

Fig. 2. Comparison of fluorescence intensity in the reactions of $\mathbf{1}$ or $\mathbf{2}$ and some amino acids (a) and a 
reaction profile of $\mathbf{1}$ or $\mathbf{2}$ and glycine (b). (a) [2] $=1.0 \mathrm{mM}$, [amino acid] $=3.0 \mathrm{mM},[B S A]=6 \mathrm{mg} / \mathrm{ml}$ in acetone-phosphate buffer (1:3) solution. (b) $[1]=[2]=1.0 \mathrm{mM}$, [glycine] $=0-3.0 \mathrm{mM}$ in acetone-phosphate buffer (1:3) solution.

To understand the observed different reactivities and fluorescent intensities of the adducts, the molecular orbital calculations were made. Fig. 3 shows the LUMOs of the three fluorescamines, in which the LUMOs are developing on the $3(2 H)$-furanone ring in all compounds. The C-5s at which the Michael addition takes place are covered by LUMOs, and the Mulliken atomic charge of C-3 and C-5 slightly increases in the order of $\mathbf{1 , 2}$, and $\mathbf{3}$, which is in agreement with the observed reactivity toward glycine as described above. The introduction of cyano groups affected the electronic distribution of C-5 slightly greater than that of C-3 carbonyl carbon. In addition, the emission energies of the HOMO-LUMO transitions for the 1, 2, and 3- $\mathrm{MeNH}_{2}$ adducts as the concise models were calculated to support the observed difference in the fluorescence properties, the calculations giving the energies as $3.39,3.63$, and $3.57 \mathrm{eV}$, respectively, which explains the above observed blue-shift of 2 - and 3-phenylalanine adducts compared to the adduct of $\mathbf{1}$.

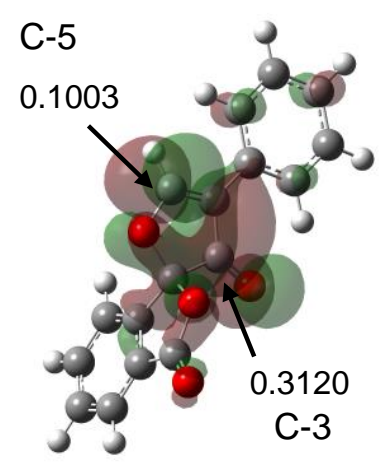

1

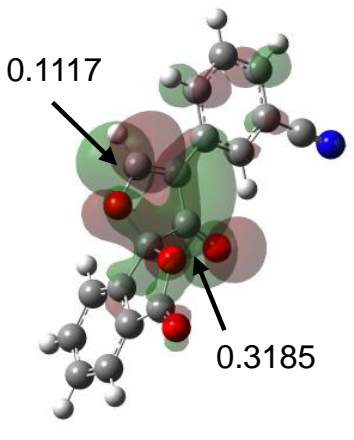

2

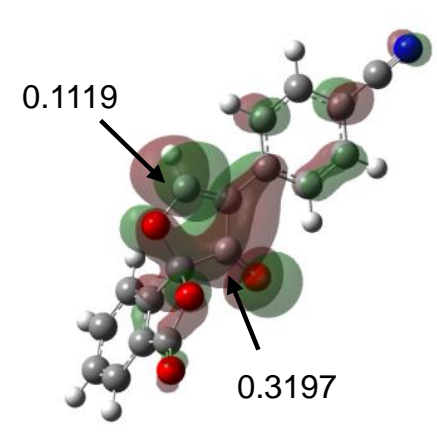

3

Fig. 3 LUMOs of fluorescamine 1 and its derivatives $\mathbf{2}$ and 3, and the Mulliken atomic charges at C-3 and C-5 calculated by Gaussian 09 at the B3LYP/6-31G level.

Many fluorescent compounds can be used as the fluorophores for the peroxyoxalate chemiluminescence (PO-CL), which takes place by the interaction of the high-energy intermediate, 
generated by the reaction of an active oxalate and hydrogen peroxide, and a fluorescent compound as an energy acceptor. ${ }^{11}$ Therefore, the fluorescent adducts of fluorescamines and amino acids described above are also applicable for this chemiluminescence system. In spite of increasing investigations of the chemiluminescent detection of amino acids, the fluorescamine-amino acid adducts have been, to our knowledge, never used for the chemiluminescence systems until now. ${ }^{12}$ The numbers of photons produced by adding an aqueous solution of alkaline hydrogen peroxide (Solution A) to a solution containing 1 or $\mathbf{2}$, amino acids, and bis(4-chlorophenyl) oxalate in THF/water (1:1) (Solution B) were counted by a photomultiplier tube, and the chemiluminescence quantum yields $\left(\Phi_{\mathrm{CL}}\right)$ were estimated as shown in Fig. 4. The capability of the amino acid detection by the chemiluminescence system was demonstrated and distinct preference of $\mathbf{2}$ over $\mathbf{1}$ was also observed, in which all the $\Phi_{C L}$ for $\mathbf{2}$ are larger by 2-5 times than those for $\mathbf{1}$ in all cases of the measurements using four amino acids. The larger enhancement of the chemiluminescence intensities compared to that observed in the fluorescence measurements are probably due to the different excitation processes among them. The cyano-substituent in the fluorescent adducts would affect the chemiluminescence reactions favorably in the reaction field, but further details are unclear at present. Comparing to the fluorescence method measureable at picomole level of amino acids, the chemiluminescence quantum yields were very low even at micromolar level of the concentrations in our photon-counting system. However, much higher sensitivity is expectable, if the chemiluminescence analytical system is suitably prepared. ${ }^{13}$

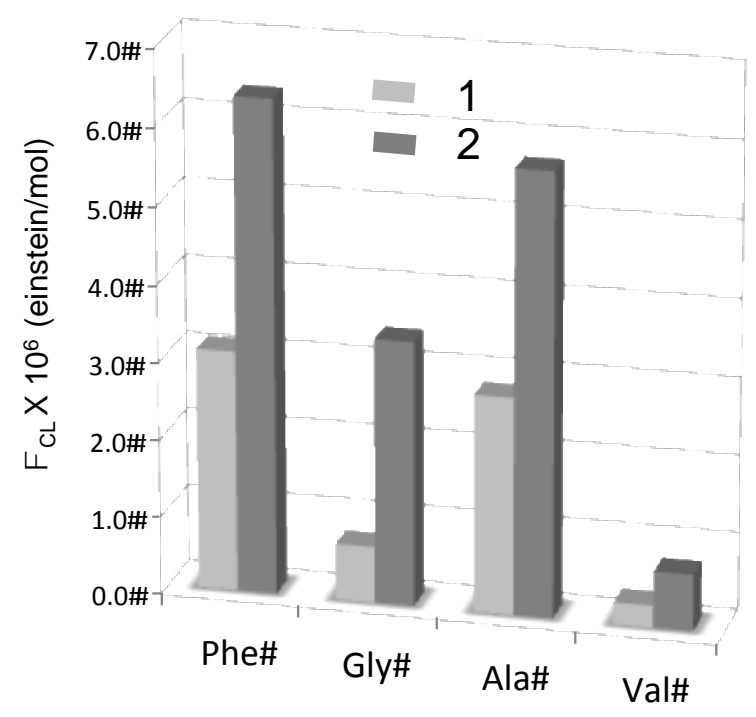

Fig. 4. Comparison of peroxyoxalate chemiluminescence quantum yields for the adducts of $\mathbf{1}$ or $\mathbf{2}$ and amino acids. Solution $\mathrm{A}:\left[\mathrm{H}_{2} \mathrm{O}_{2}\right]=2.5 \times 10^{-2} \mathrm{M},\left[\mathrm{Na}_{2} \mathrm{CO}_{3}\right] 1.0 \times 10^{-3} \mathrm{M}$ in water. Solution B: 
$[1]=[2]=7.5 \times 10^{-4} \mathrm{M}$, [amino acid $]=1.5 \times 10^{-3} \mathrm{M}$, [bis(4-chlorophenyl) oxalate $]=7.5 \times 10^{-5} \mathrm{M}$ in THF/water (1:1) solution.

Consequently, a new cyano-substituted fluorescamine derivative (2) provides preferential properties, such as the higher reactivity toward amino acids and the stronger fluorescence intensities of the adducts, comparing with the original fluorescamine (1). It is expected that 2 will be used for the amino acid and protein analyses in place of the original 1.

\section{Supplemental data}

Supplemental data associated with this article can be found in the online version at http://

\section{References}

1. (a) Udenfriend S, Stein S, Boehlen P, Dairman W, Leimgruber W, Weigele M. Science. 1972; 178: 871-872. (b) Weigele M, DeBernard SL, Tengi JP, Limgriber W. J. Am. Chem. Soc. 1972; 94 : $5927-5928$.

2. (a) Zhou Y, Yoon J, Chem. Soc. Rev. 2012; 41: 52-67; Fig.

(b) Toyo'oka, T. Chromatography, 2012, 33, 1-17.

3. (a) Pravadali-Cekic S, Jones A, Kazarian AA, Paull B, Soliven A, Ritchie H, Camenzuli M, Dennis GR, Shalliker RA. Microchem. J. 2015; 121: 141-149;

(b) Karasakal A, Tatar ULUS, Unal DO. Optics and Spectroscopy, 2015; 119: 318-321;

(c) Rageh AH, Klein KF, Pyell U. Chromatographia, 2016; 79: 225-241;

(d) Omar MA, Hammad MA, Salman BI, Derayea SM. Spectrochim. Acta. A: Mol. Biomol. Spectroscopy, 2016; 157: 55-60;

(e) Ashby J, Duan Y, Ligans E, Tamsi M, Zhong W. Anal. Chem. 2015; 87: 2213-2219.

4. Stein S, Boehlen P, Stone J, Dairman W, Udenfriend S. Arch. Biochem. Biophys. 1973; 155 : 203-212.

5. (a) Leimgruber W, Weigele M. Ger. Offen. 1973; DE 2263534 A1 19730705; 
(b) Leimgruber W, Weigele M. Ger. Offen. 1974; DE 2350179 A1 19740502;

(c) Leimgruber W, Weigele M. US Patent 3917646 A 19751104;

(d) Leimgruber W, Weigele M. US Patent 3957826 A 19760518.

6. Weigele M, Tengi JP, DeBernard S, Czajkowski R, Leimgruber. J. Org. Chem. 1976; 41: 388-389.

7. (a) Anwar S, Li SM, Chen K. Org. Lett. 2014; 16: 2993-2995;

(b) Zhao B, Liang HW, Yang JYZ, Wei Y. Catalysis. 2017; 7: 5612-5617.

8. Weigele M, DeBernardo SL, Tengi JP, Leimgruber W. J. Am. Chem. Soc. 1972; 94:5927-5928.

9. Stein S, Boehlen P, Udenfriend S. Arch. Biochem. Biophys. 1974; 163:400-403.

10. Chen RF, Smith PD, Maly M. Arch. Biochem. Biophys. 1978; 189: 241-250.

11. (a) Maruyama T, Narita S, Motoyoshiya J. J. Photochem. Photobiol. A: Chemistry. 2013; 252: 222-231;

(b) Ciscato LFM, Augusto FA, Weiss D, Bartoloni FH, Albrecht S, Brandl H, Zimmermann T, Baader WJ. Arkivoc. 2012; 391-430.

12. Zacharis CK, Tzanavaras PD. Anal. Chim. Acta. 2013; 798: 1-24.

13. (a) Zhu R, Kok WT. J. Pharm. Biomed. Anal. 1998; 17: 985-999;

(b) Tsunoda M, Imai K. Anal. Chim. Acta. 2005; 541: 12-23;

(c) Garcia-Campana, AM, Lara FJ. Anal. Bioanal. Chem. 2007; 387: 165-169. 